\title{
Blogging Climate Change: A Case Study
}

\author{
Erangu Purath Mohankumar Sajeev, Kian Mintz-Woo, \\ Matthias Damert, Lukas Brunner and Jessica Eise
}

\begin{abstract}
Public perception of the magnitude of challenges associated with climate change is still lower than that of the majority of scientists. The societal relevance of climate change has raised the need for a more direct communication between scientists and the public. However, peer-reviewed scientific articles are not well-suited to engaging a wider audience. This begets a need to explore other avenues for communicating climate change. Social media is a vibrant source for information exchange among the masses. Blogs in particular are a promising tool for disseminating complex findings on topics such as climate change, as they are easier to comprehend and are targeted at a broader audience compared to scientific publications. This chapter discusses the usefulness of blogs in communicating climate change, using our blog Climate Footnotes (climatefootnotes.com) as a case study. Drawing from communication theory and our experiences with Climate Footnotes, we identify and describe elements such as message framing, translation of scientific data, role of language, and interactivity in aiding climate change communication. The insights outlined herein help understand the nature and impact of online climate change communication. The chapter may also serve as a useful blueprint for scientists interested in utilizing blogs to communicate climate change.
\end{abstract}

\section{Introduction}

While the impacts of anthropogenic climate change are constantly increasing (Fischer and Knutti 2015), the same cannot be said for the public's concern. Climate change is a slow phenomenon. Manifesting over years and decades, it lacks

E. P. M. Sajeev $(\square)$

Rothamsted Research, AL5 2JQ Harpenden, UK

e-mail: em.sajeev@rothamsted.ac.uk

E. P. M. Sajeev · K. Mintz-Woo · M. Damert · L. Brunner

DK Climate Change, University of Graz, Graz 8010, Austria

J. Eise

Brian Lamb School of Communication, Purdue University, West Lafayette, IN 47907, USA

(C) The Author(s) 2019

W. Leal Filho et al. (eds.), Addressing the Challenges in Communicating Climate

Change Across Various Audiences, Climate Change Management,

https://doi.org/10.1007/978-3-319-98294-6_9 
the timeliness and punch of so many of today's headliners and hot-button issues of concern. It is a uniquely difficult challenge insofar as it requires people to act with urgency to prevent future scenarios that many find unimaginable or even unbelievable in their present condition. As such, human interest in climate change has been slow to garner and difficult to maintain.

Yet despite this challenge, public interest in and perception of climate change is critical. Without an informed public, governments, scientists and organizations will lack the social license necessary to act decisively to mitigate the impacts of this phenomenon. What is more, individuals who may wish to take action in their personal lives to lower their carbon footprint may lack the resources to do so or even the understanding that doing so is a decision that they could make.

Public perception of the magnitude of challenges associated with climate change is not where it needs to be. In a 2016 study conducted in a sample of countries in Europe, $16 \%$ of Germans and $14 \%$ of British were sceptical about human activity as a cause for climate change or did not believe that climate change occurs, and on an open-ended question less than $3 \%$ in both countries listed "climate change" as one of the toughest challenges their country will face in the next 20 years (Steentjes et al. 2017). In the United States, over half of Americans do not believe that climate change is due to human activity. A 2016 Pew Research study reports that roughly three-in-ten say it is due to natural causes $(31 \%)$ and another fifth say there is no solid evidence of warming (20\%) (Funk and Kennedy 2016). However, the same 2016 Pew Research study finds that a large majority (67\%) want climate scientists to have a major role in climate policy - the most supported group considered-and a larger majority (78\%) trust climate scientists "some" or "a lot" to provide accurate climate information. The public believes climate scientists have roles both in policy and in communication. As such, engaged participants in climate change-and scientists in particular-might assume responsibility for conducting outreach around the issue themselves. What is more, when it comes to issues with high societal relevance, researchers have an obligation to help inform a more general audience about their findings and associated implications (Leshner 2003).

\section{Blogs as a New Medium of Climate Change Communication}

The primary method of communication and dissemination of scientific findings has been and continues to be peer-reviewed scientific articles. While this method is uniquely suited to communicating to an academic audience, ensuring rigor and accuracy of scientific findings, it is less appropriate for a wider audience. Many aspects of peer-reviewed scientific articles make them a poor choice for communicating with a broader audience, aspects ranging from paywalls and jargon to (frequently) dense writing styles. 
These are not the only challenges associated with the peer-reviewed scientific model of communication. While the scientific community has recognized the importance of disseminating information to the public, many still operate under the assumption that the critical barrier to communication is the lack of information that exists among the public. Hence a lot of effort around science communication is focussed on providing more information on the basis that once the public understands the science; they would be more inclined to agree with science and to endorse action. However, communication theory suggests otherwise and lists factors such as interactivity, inclusivity, trust and social identity as having stronger impacts than providing information alone (Bubela et al. 2009). Hence, merely amplifying exactly what is in the peer-reviewed scientific literature as many times as possible with the assumption that people will eventually understand and take desired action is too simplistic.

These communication challenges beget the need to explore new avenues for exchanging information. With the advent and progress of the technological era, there has been a shift from traditional modes of communication that employ a one-to-many communication strategy to a more interactive form of communication that uses a many-to-more strategy. Today's online communication environment, Web 2.0, is "a collaborative medium that allows users to communicate, work together and share and publish their ideas and thoughts" (Rollett et al. 2007). Much of that communication and collaboration occur across social media, a communication avenue that has emerged as a vibrant source for information exchange in society. One among the many promising new communication avenues that have emerged are blogs. Unlike traditional communication channels, blogs offer a platform to the users where information is interactive and inclusive irrespective of geography and socio-economic standing (O’Neill and Boykoff 2011).

According to the internet use statistics for 2017, almost 52\% of the world population are internet users with a growth rate of $976 \%$ in the last 7 years. ${ }^{1}$ According to the Pew Research Center, ${ }^{2}$ a survey conducted in 2016 reveals that around $38 \%$ of Americans get their news online (via mobile or desktop), easily dwarfing legacy communication channels such as the radio (25\%) and print media $(20 \%)$. A breakdown by age group indicates online sources as the dominant medium of consumption of news for people below 50 years of age. As of 2012, there were 50 million blogs and this is expected to rise as numbers of users and blog posts on popular blogging platforms such as Tumblr, Wordpress and Blogger are increasing. Regarding science blogs, earlier estimates were in the range of 1000 1200 (Trench 2012). A review study by Schäfer (2012) estimates the number of science blogs to be about 1900 of which 1400 were "climate" blogs with only 323 actually categorized as "climate science" blogs. Even accounting for the existence of psuedoscience blogs folded into that tally, 1900 blogs compared to the 50 million that currently exist makes that number paltry indeed. As a form of new media, blogs

\footnotetext{
${ }^{1}$ http://www.internetworldstats.com/stats.htm.

${ }^{2}$ http://www.journalism.org/2016/07/07/the-modern-news-consumer/.
} 
are part of the worldwide communication network and thus accessible "to diverse geographical and socio-economic populations, which provides new opportunities for engaging individuals with climate change" (O’Neill and Boykoff 2011). Given the potential of the internet and social media, the resources of Web 2.0 are tools that ought not to be ignored in the quest for wider outreach.

One of main functions of Web 2.0 is as 'places to learn' for formal and non-formal education as well as informal learning (Schugurensky 2000). Adult learners often benefit by optimizing these web-based spaces as alternative environments for informal learning when they navigate through information, network with other people, or produce wanted identities (Selwyn 2007). Informal learning is any activity involving the pursuit of understanding, knowledge or skill that happens without externally imposed curricula (Livingstone 2001). Informal learning is important for adults because it highlights the learner as an agent of learning and also since it happens in everyday life, it expands the conventional meaning of learning (Livingstone 2001; Marsick and Watkins 2001). With this perspective of online spaces, social media is not just a space for entertainment but also informal learning. Adult learners can determine their roles and degrees of engagement in the activities found in these web-based spaces. More interactive ways of using Web 2.0 may guarantee not only more diverse, but also better quality learning (Heo and Lee 2013).

Blogs in particular are a promising tool for disseminating complex findings on topics such as climate change for a number of reasons. Web 2.0 is a medium that allows users to share and make public their ideas and thoughts with no barrier to entry beyond an internet connection and literacy. Researchers are often hesitant to put forward their findings as they are concerned that they may not be properly validated or posited. Yet in most online spaces, no one else shares those qualms. Blogs are a good way for researchers to get over this and join the fray. Blogs necessitate neither the brevity nor the pith of a 140-character tweet, yet remain an informal and easily-accessible mode of communication for the broader public. They make for good training wheels, so to speak. Researchers can use blogs as a platform to engage with a broader audience allowing for collaboration and networking due to the quick and timely discussions that can take place. As far as young researchers are concerned, blogs serve as a testing ground to improve their writing skills, build an online identity and provide a learning opportunity by exposing them to new perspectives and the latest advances in technology and research (Putnam 2011). What is more, science to public communication is viewed as helpful for the process of conducting science as it can assist in generating ideas and disseminating policy proposals (Blanchard 2011). Academic journals, which traditionally used to communicate only via peer-reviewed articles, have also taken a step in the direction of new media. Examples include blogs by powerhouses of academic publishing such as Nature, Science, and Cell (Blanchard 2011).

In addition, topics around climate change are complex. This makes blogs an appropriate medium over other social media options. Blogs allow for depth and range. A blog can be as short as a couple hundred words or as long as several thousand (although best practices would suggest you should cap your length to around 400-700 words per entry). While videos are compelling, it is unreasonable 
to expect researchers to become videographers. It is less unreasonable to expect that researchers write. In addition, a blog may be contributed to as a 'guest' writer and could be a periodic activity in which a scientist might engage, not something he or she is forced to maintain on a daily basis such as Twitter or Instagram. Perhaps more to the point, however, blogs have the potential to reach a broad audience beyond the confines of academia and research communities. They allow for timely publication on issues as there is no review lag time, making it more interesting and engaging for a public readership.

The direct and rapid outreach of scientific information through new communication channels such as blogs creates a means for engagement and participation for readers and writers alike. For these reasons, we examine the use of blogs to disseminate scientific information on the complex phenomenon of climate change. In this chapter, we shed light on effective strategies, approaches and limitations using our own blog Climate Footnotes (climatefootnotes.com) ${ }^{3}$ as a case study. We believe these insights may help in understanding how information related to climate change can be disseminated through online platforms such as blogs. This type of understanding of public engagement helps build the knowledge base necessary for a holistic approach encouraging the interplay between the scientists and the public in addressing topics related to climate change.

\section{Our Blog-Climate Footnotes}

While there are climate blogs that aim to provide timely climate scientific context (e.g., www.RealClimate.org) and projects that aim to provide attribution analyses for particular extreme weather events (e.g., www.climatecentral.org/), we created our blog motivated by the desire to increase the ranks of graduate students in scientific blogging (Wilkins 2008). Climate Footnotes serves as a space to discuss our scientific ideas and achievements as well as address personal and political topics. Different members of the Doctoral College Climate Change (DKCC) ${ }^{4}$ at the University of Graz participated on a voluntary basis, with a strong majority of the students contributing at least once. As of October 2017 more than 70 posts have been published on the blog on a more or less regular basis. We ultimately settled on a posting schedule of twice per month with a rotating schedule of students, which proved to be a reasonable expectation measured against the pressures of other academic work. We also periodically invited guest bloggers to participate.

An important component of our success is an automated reminder system that allows contributors to publish on their own time schedule with only a small role for editors. Initially, editors spent a lot of energy contacting people and arranging for

\footnotetext{
${ }^{3}$ Throughout, when referring to particular blogposts on Climate Footnotes, we simply put the blogpost title in quotation marks.

${ }^{4}$ http://dk-climate-change.uni-graz.at/en/.
} 
comments. This proved untenable and ultimately the amount of editing needed was limited. We ultimately settled on "editing as optional" although there is constant transparency and editing access (an email address is dedicated to blogposts in progress). Everyone has individual accounts which they can use to self-publish. We elected to publish in a private forum-i.e. not on the university system - as it afforded more control and no restriction in terms of content and we paid for the domain registration privately. Moreover, modern publishing platforms make setting up a blog rather easy, for Climate Footnotes we used a basic WordPress (https:// wordpress.org/) blog template, which provides all of the required functionality.

Before starting with Climate Footnotes, the team behind the project decided not to target a specific audience or limit contributions to specific aspects of climate change. Behind this decision were two main considerations, one theoretical and one functional. First, as explained above, blogs are theoretically accessible for a large amount of people around the globe and although its impacts may vary, climate change represents a challenge that is not restrained to specific regions or groups in society. Second, in order to benefit from the interdisciplinary composition of the team of authors, we did not want to define a specific scope of topics. We view our blog as an outlet that provides us with the opportunity to creatively experiment with different formats of texts and writing styles to convey scientific messages. The underlying rationale was the wish to understand how different formats appeal to readers and why. From our point of view, this openness and potential for creative freedom would have been impeded by limiting the scope of the blog. The different contributors provided a breadth of styles, which benefitted the blog without making it feel fragmented.

\section{Our Experience with Blogging Climate Change}

In terms of geographical coverage, our website statistics illustrate that people reading our blog are primarily from German or English-speaking countries (see Fig. 1). Almost $43 \%$ of the readers were from Austria. This is probably due to the fact that the team of authors is mainly based in Austria, which facilitates word-of-mouth advertising in this region. Users from Germany also comprised around $8 \%$ of the readership due to a few German blog posts and bloggers from Germany. However, due to the primary language of published articles being English readers from English speaking countries such as the United States, Canada and United Kingdom or countries that operate in English academically like India and South Korea were also prevalent.

In the following sections, we critically discuss our experiences with regard to drivers and barriers to successful climate communication via weblogs. Based on the website statistics of Climate Footnotes and discussions with colleagues and readers, we have identified the following main factors that can further help blogs in effective communication: 


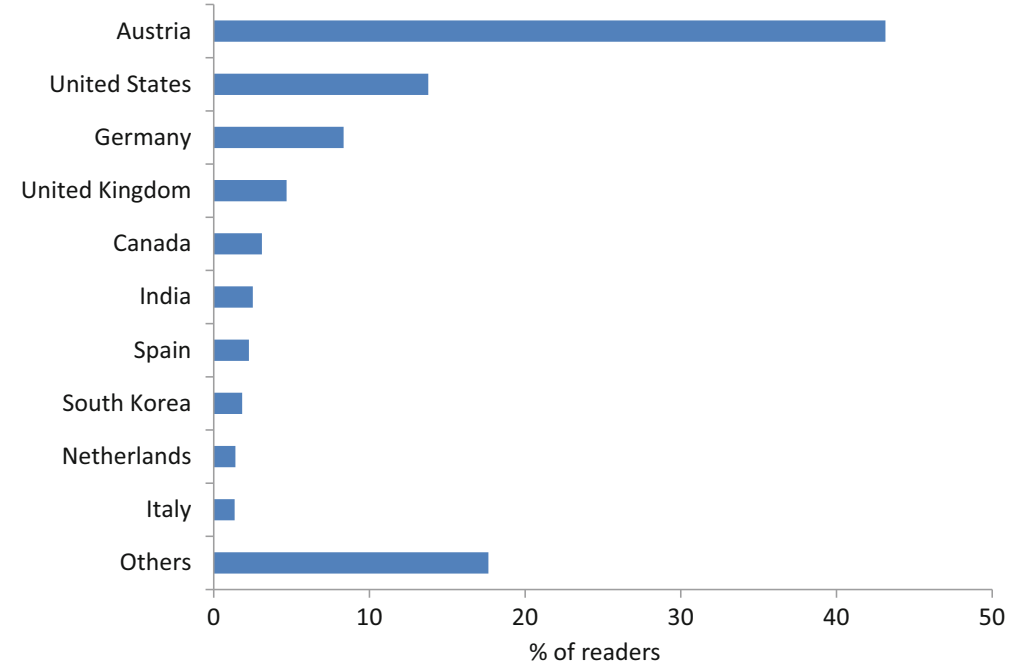

Fig. 1 Geographical distribution of readers

1. Breaking down complex scientific results into commonly understandable language (Sect. 4.1)

2. Responding to recent major events or articles that have recently been published in popular newspapers or magazines and addressing topics that are controversially discussed in a climate change context (Sect. 4.2)

3. Relating posts to people's everyday life, e.g., by means of storytelling or referring to specific regional and local contexts (Sects. 4.3 and 4.5)

4. Including interactive contents (Sect. 4.4)

5. Discussing weblog ethics and the possibility of controversial or dubious research (Sect. 4.6)

6. Promoting the blog through social media (Sect. 4.7).

\subsection{Translating Scientific Data into Simple Terms}

Results of scientific research are often solely published in peer-reviewed journals aimed at a small sub-group of the scientific community. But keeping in touch with a broader audience can help scientists reflect on their work and objectives (Dudo and Besley 2016). Also, in publicly funded research, an argument can be made for an obligation to communicate the results to the society (Lubchenco 1998), and when it comes to topics with a high societal relevance such as climate change and its implications, communication and interaction with society becomes even more crucial (Bonetta 2007). 
In our blog, several posts break down topics connected to climate change, ranging from very broad questions like "Who measures global temperature?" to quite specific ones, like "Cheese versus Meat-Which Carbon footprint is higher?" The challenge of communicating a counterintuitive scientific concept also surfaces at times, such as with the post "Why is the pressure in meter?" This post originated from a long discussion of the author with the University's public relations department. In meteorology, upper-air pressure systems are frequently depicted as height anomalies (and are hence associated with the unit of length); however, for a layman these units of measurement could create confusion since pressure is usually measured in pascal or bar. In situations like this figures proved to be a powerful addition to mere text in explaining the underlying concepts and reasons. A blog provides a favourable frame for such an explanation which is uncommon in peer-reviewed scientific journal and would be at best available only when being entirely comprehensive, e.g., in a textbook.

\subsection{Providing Perspectives on Current Events}

One important advantage for blogs is that they can quickly amplify and respond to points in the news. One example which received a large amount of international public attention was the 2016 US Presidential Election. So, for instance, while it was fairly unknown what Secretary Clinton's climate policies were in the general election process, one of us wrote about what she had promised ("Clinton and Climate Change"). After the election, when Trump won unexpectedly, there was a lot of demand for discussion of potential policy implications and there was a new post on that topic ("The International Climate Effects of Trump"). By responding to potential policy implications of current events, the blog is able to participate in an interesting and important broader discussion. It is also worth introducing the science to help explain major events. For instance, after the devastating hurricane Matthew in 2016, a post addressed the question "Does global warming bring Matthew?" and also discussed the possibility of humans ever being able to stop such hurricanes.

\subsection{Role of Language}

For non-natives, the English language - much like jargon - can pose a barrier in understanding scientific text. However, even research focusing on regional and local issues is often published in international (and therefore English-language) journals making it harder for the regional (or local) community to become informed. In this case, breaking down the results in a blog post written in, for instance, German can reach more people. Some of the topics presented in our blog have a quite strong local focus (e.g., "The Raab Valley-A Catchment Description 
from a Hydrological View Point") and as Fig. 1 shows more than 50\% of our readers come from German-speaking countries. The post "The pressure is on!About the European cold spell in spring 2016"/“Nur nicht zu viel Druck! - Der Kälteeinbruch im Frühling 2016" was simultaneously published in English as well as German. The German version had almost 50\% more views, highlighting the potential of communicating local and regional results in the respective native language.

Another possibility of making scientific knowledge more comprehensible for a broad audience is storytelling. Dahlstrom (2014) points out that "research suggests that narratives are easier to comprehend and audiences find them more engaging than traditional logical-scientific communication". Embedding scientific information into a story based on personal experience or pure fiction can help readers to identify themselves better with the issue and increase feelings of empathy. This especially applies to narratives that involve certain individuals to which a reader can emotionally connect; a concept called "personification" (Dahlstrom 2014). Our blog statistics confirm this claim. One of the more popular blog posts titled "Climate conferences and flying" addresses a paradox commonly experienced in a climate scientist's everyday life: climate scientists fly more frequently than the average population and thus have a large carbon footprint. Engaging with this paradox with reference to personal experience increased the narrative value and interest. Indeed, our website statistics show that blog posts that relate to personal experiences attract more readers and we would therefore suggest to carefully use storytelling for science-to-public communication on weblogs as, e.g., in the post "Diagnosis 'Cartastrophe': depressed cars are damaging the environment". This post uses cars as protagonists in order to recontextualize the counterproductive way that cars are treated.

\subsection{Interactive Communication}

The blogging platform is designed in a way which is conducive to textual interaction, collaboration and social networking. The ability to organize information with the use of tags, categories etc. along with the use of embedded hyperlinks and even videos lends a dynamic nature and provides a greater depth to texts bringing in a diversity of voices to the fray. This allows for better access and ease of information to the readers compared to journal articles and print media (Davies and Merchant 2007). This new interactive model characterized by openness and short response time leads to the generation of a multitude of perspectives (O'Neill and Boykoff 2011). Such feedback and critiques allows for rapid development of ideas in contrast to academic journals where responses are usually incorporated towards the end stage of the publication process (Bubela et al. 2009). A survey on indicators of interactivity in academic blogs show that the level of interaction found in online blogs is similar to those at scientific conferences and meetings (Luzón 2011). Thus academic blogs can serve as an online meeting place facilitating information exchange and collaboration without the rigors of organizational issues related to 
conferences and meetings (Wulff et al. 2009). A particular post we would like to highlight in this regard was an online interactive quiz to test and spread awareness and information on climate change ("Take the Ultimate Climate Change Quiz!"). The post was created using a freely available quizzing platform called Qzzr. The quiz had ten multiple-choice questions related to climate change basics, news and politics. The quiz was the most popular post on the blog. One of the reasons for the popularity was most likely the interactive aspect, but it was also easy to share it with readers on other social media platforms which also encouraged participation.

\subsection{Focussing on Topics Close to Home}

Communication of climate change is easier when reflecting local experience (Shome et al. 2009). The cold spell which hit parts of Europe in late spring 2016 was coincidently strongly connected to one of the authors' research fields. A scientific article (Brunner et al. 2017) on the more general development of spring cold spells had been published only a few months earlier. The blog served as an ideal platform to communicate the specific meteorological condition which led to this cold spell based on the publication to a broader audience ("The pressure is on! -About the European cold spell in spring 2016").

Policy and local extreme weather events can even be connected such as in a post on Canadian forest fires ("Discussing Climate Disasters-Canada's Forest Fires"). Like Australia, Canada's economy is highly dependent on energy sources which contribute to climate change. But when forest fires hit the towns which are disproportionately responsible for contributing to the problem, there is an irony which is hard to miss. Another blogpost detailed the participation of one of the authors at the European Geosciences Union's General Assembly in Vienna ("EGU 2016 or Vienna stuffed with over 12,000 scientists"). This gave readers a first-hand narrative of a major European conference and the experience of a participating researcher. Such initiatives give the public access and a chance to appreciate the social workings of science, which is exclusive otherwise.

\subsection{Weblog Ethics}

Blogs are basically unregulated and non-peer-reviewed outlets and as such do not have strict underlying quality control. Yet, as is the case when doing research and presenting it at conferences and in scientific journals, researchers should ensure the correctness and transparency of what they publish on weblogs. Blood (2002) provides useful suggestions for universal weblog ethics and summarizes them in the following six points: 
(1) one should publish as fact only that one believes to be true;

(2) if the material one refers to exists online, link to it;

(3) one should publicly correct any misinformation;

(4) one should write each article as if it could not be changed; one can add to it, but should not rewrite or delete, any entry;

(5) one should disclose any conflict of interest; and

(6) state questionable or biased sources.

When looking at these guidelines, it becomes obvious that most of them are also relevant as recommendations for best-practice research. In contrast to scientific publications, however, one major difference is worth highlighting: researchers might want to deliberately discuss biased or even dubious sources in their blog posts. Although there is danger of misleading communication if such sources are not acknowledged, a blog post can serve as a valuable platform for confronting questionable studies and their results with a researcher's personal opinion or other (reliable and transparent) scholarly work.

This especially applies to the climate change context. In order to tackle climate denial, for example, recent research argues that "normal academic response does not work since it presupposes that both parties follow basic rules of rational argumentation such as looking at the evidence as a whole" and that "denialism cannot be defeated by just providing more information" (Björnberg et al. 2017). In contrast to academic journals, which are mostly subject to these limitations, blogs can provide researchers with the opportunity to openly reflect on such issues and react to current developments with only minimal delay (see Sect. 4.2). Due to the more liberal format, blogs can thus also be used to transfer a (purely scholarly) discussion from the literature to a broader audience.

\subsection{Readership and Promotion}

To increase readership and target a wide range of audiences, we tried to promote our blog through different communication channels, such as Facebook, university homepages, academic conferences or mailing lists. Interestingly, however, most readers seem to have accidentally "stumbled upon" our blog while searching the internet for specific terms. Our website statistics show that search engines were the most common referrers ( $82 \%$ of clicks), indicating that is important to include relevant keywords in blog posts and use search engine optimization for webpages.

Facebook proved to be the second most common referrer ( $6 \%$ of clicks), which shows that sharing new blog posts on social networks with friends and acquaintances is an important factor for knowledge dissemination. It should be noted that Twitter might be another useful communication outlet. The integration of Twitter into our communication strategy, however, is still in the planning phase and at this time little can be said about its potential benefits. 
In general, the challenge of spreading word of our blog was one of the most difficult issues we faced. While a few blog posts gained traction, the most popular was only seen by a few hundred people, sobering statistics for the amount of time invested (despite being a number far greater than the average academic journal article). While it does take time for blogs to gain momentum and traction (search engine optimization can take years, audiences time to acclimate to a new source, differentiation with competition is timely) and therefore success remains undefined for some time, in the end the primary motivation has been what little communication we can facilitate, a sense of obligation as scientists to contribute to a public debate and the challenge of stretching our capacities and improving our writing skills.

\section{Conclusions}

Social media is a viable and effective medium to disseminate information about climate change. However, the field would benefit from substantially more research on the use and implications of social media concerning climate change. Consequently, analysis of the various facets related to online climate change communication is relevant and requisite. This study describes the potential of one such medium of online communication - blogs - using our own experience with Climate Footnotes as a case study. Through our experience and a review of relevant literature, we highlight the usefulness of blogs in providing scientific education, furthering scientific discussions and encouraging participatory science. Furthermore, drawing from communication theory and our experience, we also identify key factors that may help researchers utilize blogs to communicate issues associated with climate change effectively across a wider audience such as:

- Making information memorable and impactful by reducing scientific complexity

- Quickly addressing and responding to current events that are in the news

- Using experiential and emotional frames like stories and personal experiences

- Tapping into the interactive potential of blogs using hyperlinks, comments and other features

- Fostering interest of the public using a regional or local message framing

- Ensuring proper research conduct and transparency

- Using other social media platforms to share and market the blog.

We believe that the ideas outlined here could be helpful for researchers who are trying or considering trying blogs as a medium to communicate climate change science. However, while the potential of scientific blogging is apparent, it has not yet been fully realized and our blogging experiences are not necessarily representative. It would be beneficial for other scientific blogging practitioners to continue to publish their experiences so that we can collectively determine best practices. This calls for further research and initiatives to deepen our understanding of the 
nature and impact of online climate change communication outlets, including blogs. This will enable the scientific community to explore how we might leverage our voice in the conversation around the pressing global challenge of climate change.

Acknowledgements The authors want to thank all other colleagues who also were part of the blogging team for valuable contributions and discussions, namely Arijit Paul, Sungmin O, Eike Düvel, Johannes Haas, Clara Hohmann, Philipp Babcicky, Javier Lopez Prol, Vincent Heß, Micheal Kriechbaum and Daniel Petz. This work was funded by the Austrian Science Fund (FWF) under research grant W 1256-G15 (Doctoral Programme Climate Change-Uncertainties, Thresholds and Coping Strategies). L. Brunner was financially supported by a Marietta Blau Grant by the Austrian Exchange Service (OeAD), financed by funds from the Austrian Federal Ministry of Science, Research and Economy (BMWFW).

\section{References}

Björnberg KE, Karlsson M, Gilek M, Hansson SO (2017) Climate and environmental science denial: a review of the scientific literature published in 1990-2015. J Clean Prod 167:229-241

Blanchard A (2011) Science blogs in research and popularization of science: why, how and for whom? In: Cockell M, Billotte J, Darbellay F, Waldvogel F (eds) Common knowledge: the challenge of transdisciplinarity. EPFL, Lausanne, pp 219-232

Blood R (2002) "Weblog ethics", the weblog handbook: practical advice on creating and maintaining your blog. Perseus Publishing, Cambridge, pp 114-121

Bonetta L (2007) Scientists enter the blogosphere. Cell 129(3):443-445

Brunner L, Hegerl GC, Steiner AK (2017) Connecting atmospheric blocking to European temperature extremes in spring. J Clim 30(2):585-594

Bubela T, Nisbet MC, Borchelt R, Brunger F, Critchley C, Einsiedel E et al (2009) Science communication reconsidered. Nat Biotechnol 27(6):514-518

Dahlstrom MF (2014) Using narratives and storytelling to communicate science with nonexpert audiences. Proc Natl Acad Sci 111(Supplement 4):13614-13620

Davies J, Merchant G (2007) Looking from the inside out: academic blogging as new literacy. A New Literacies Sampler pp 167-197

Dudo A, Besley JC (2016) Scientists' prioritization of communication objectives for public engagement. PLoS ONE 11(2):e0148867

Fischer EM, Knutti R (2015) Anthropogenic contribution to global occurrence of heavy-precipitation and high-temperature extremes. Nat Clim Change 5(6):560-564

Funk C, Kennedy B (2016) Public views on climate change and climate scientists. Retrieved 08 Sept, 2017. From http://www.pewinternet.org/2016/10/04/public-views-on-climate-changeand-climate-scientists/

Heo GM, Lee R (2013) Blogs and social network sites as activity systems: exploring adult informal learning process through activity theory framework. Educ Technol Soc 16(4):133-145

Leshner AI (2003) Public engagement with science. Science 299(5609):977

Livingstone DW (2001). Adults' informal learning: definitions, findings, gaps, and future research. NALL Working Paper\# 21

Lubchenco J (1998) Entering the century of the environment: a new social contract for science. Science 279(5350):491-497

Luzón MJ (2011) 'Interesting post, but I disagree': social presence and antisocial behaviour in academic weblogs. Appl Linguist 32(5):517-540

Marsick VJ, Watkins KE (2001) Informal and incidental learning. New Dir for Adult Contin Educ 2001(89):25-34 
O'Neill S, Boykoff M (2011) The role of new media in engaging the public with climate change. In: Whitmarsh L, O'Neill S, Lorenzoni I (eds) Engaging the public with climate change: behaviour change and communication. Routledge, London, pp 233-251

Putnam L (2011) The changing role of blogs in science information dissemination. Issues Sci Technol Librariansh 65(4)

Rollett H, Lux M, Strohmaier M, Dosinger G, Tochtermann K (2007) The Web 2.0 way of learning with technologies. Int J Learn Technol 3(1):87-107

Schäfer MS (2012) Online communication on climate change and climate politics: a literature review. Wiley Interdiscip Rev: Clim Change 3(6):527-543

Schugurensky D (2000) The forms of informal learning: Towards a conceptualization of the field. NALL Working Paper \#19, 2000. Cardiff: Cardiff University. From https://tspace.library. utoronto.ca/bitstream/1807/2733/2/19formsofinformal.pdf

Selwyn N (2007). Web 2.0 applications as alternative environments for informal learning-a critical review. Paper presented at the OECD-KERIS expert meeting - session 6-alternative learning environments in practice: using ICT to change impact and outcomes. From http:// www.oecd.org/dataoecd/32/3/39458556.pdf

Shome D, Marx S, Appelt K, Arora P, Balstad R, Broad K et al (2009) The psychology of climate change communication: a guide for scientists, journalists, educators, political aides, and the interested public

Steentjes K, Pidgeon NF, Poortinga W, Corner AJ, Arnold A, Böhm G et al (2017). European perceptions of climate change (EPCC): topline findings of a survey conducted in four European countries in 2016

Trench B (2012) Scientists' blogs: Glimpses behind the scenes. In: Rödder S, Franzen M, Weingart P (eds) The sciences' media connection-public communication and its repercussions. Springer, Heidelberg, pp 273-289

Wilkins JS (2008) The roles, reasons and restrictions of science blogs. Trends Evol Ecol 23(8): 411-413

Wulff S, Swales JM, Keller K (2009) "We have about seven minutes for questions": the discussion sessions from a specialized conference. Engl Specif Purp 28(2):79-92

Open Access This chapter is distributed under the terms of the Creative Commons Attribution 4.0 International License (http://creativecommons.org/licenses/by/4.0/), which permits use, duplication, adaptation, distribution and reproduction in any medium or format, as long as you give appropriate credit to the original author(s) and the source, a link is provided to the Creative Commons license and any changes made are indicated.

The images or other third party material in this chapter are included in the work's Creative Commons license, unless indicated otherwise in the credit line; if such material is not included in the work's Creative Commons license and the respective action is not permitted by statutory regulation, users will need to obtain permission from the license holder to duplicate, adapt or reproduce the material. 